\title{
BMJ Open Comparative efficacy and safety of different regimens of ranibizumab for neovascular age-related macular degeneration: a network meta-analysis of randomised controlled trials
}

Xinyu Zhao, ${ }^{1,2}$ Lihui Meng, ${ }^{1,2}$ Youxin Chen (D) ${ }^{1,2}$

To cite: Zhao X, Meng $L$, Chen $Y$. Comparative efficacy and safety of different regimens of ranibizumab for neovascular age-related macular degeneration: a network meta-analysis of randomised controlled trials. BMJ Open 2021;11:e040906. doi:10.1136/ bmjopen-2020-040906

- Prepublication history and additional material for this paper is available online. To view these files, please visit the journal online (http://dx.doi.org/10. 1136/bmjopen-2020-040906)

Received 25 May 2020 Revised 03 August 2020 Accepted 22 November 2020

D Check for updates

(c) Author(s) (or their employer(s)) 2021. Re-use permitted under CC BY-NC. No commercial re-use. See rights and permissions. Published by BMJ.

${ }^{1}$ Department of Ophthalmology, Peking Union Medical College Hospital, Chinese Academy of Medical Sciences, Beijing, China ${ }^{2}$ Key Laboratory of Ocular Fundus Diseases, Chinese Academy of Medical Sciences, Beijing, China

Correspondence to Dr Youxin Chen; chenyx@pumch.cn

\section{ABSTRACT}

Objective To give a comprehensive efficacy and safety ranking of different therapeutic regimens of ranibizumab for neovascular age-related macular degeneration (nAMD).

Design A systematic review and network metaanalysis.

Methods The PubMed, Embase, Cochrane Central Register of Controlled Trials, and other clinical trial registries were searched up to 1 October 2019 to identify related randomised controlled trials (RCT) of different regimens of ranibizumab for $\mathrm{nAMD}$. The primary efficacy outcome was the changes of best-corrected visual acuity (BCVA) at 1 year, the primary safety outcome was the incidence of severe ocular adverse events. Secondary outcomes such as changes of central retinal thickness (CRT) were evaluated. We estimated the standardised mean difference (SMD), ORs, 95\% Cls, the surface under the cumulative ranking curves and the mean ranks for each outcome using network meta-analyses with random effects by Stata 14.0 .

Results We identified 26 RCTs involving 10821 patients with nAMD randomly assigned to 21 different therapeutic regimens of ranibizumab or sham treatment. Ranibizumab $0.5 \mathrm{mg}$ (treat and extend, T\&E) is most effective in terms of changes of BCVA (letters, SMD $=21.41,95 \% \mathrm{Cl} 19.86$ to 22.95) and three or more lines of BCVA improvement (OR=2.83, 95\% Cl 1.27 to 4.38). However, it could not significantly reduce retreatment times compared with monthly injection (SMD $=-0.94,95 \% \mathrm{Cl}-2.26$ to 0.39$)$. Ranibizumab $0.5 \mathrm{mg}$ (3+pro re nata)+non-steroidal anti-inflammatory drugs (NSAIDs) is most effective in reducing CRT and port delivery system of ranibizumab $(100 \mathrm{mg} / \mathrm{mL})$ could reduce the number of retreatment most significantly. All regimes have no more risk of severe ocular complications (including vitreous haemorrhage, rhegmatogenous retinal detachment, endophthalmitis, retinal tear and retinal pigment epithelium tear) or cardiocerebral vascular complications.

Conclusions Ranibizumab $0.5 \mathrm{mg}$ (T\&E) is most effective in improving the visual outcome. The administration of topical NSAIDs could achieve additional efficacy in CRT reduction and visual improvement. Both interventions had acceptable risks of adverse events.
Strengths and limitations of this study

- To the best of our knowledge, this is the most comprehensive network meta-analysis which includes all the available data of randomised controlled trials and evaluates different aspects of all therapeutic regimens of ranibizumab.

- The safety and anatomic outcomes were assessed by network meta-analysis for the first time.

- As no significant inconsistency or heterogeneity was detected in our analysis, we did not perform sensitivity analysis or subgroup analysis.

- The follow-up period was limited to 12 months. The prognosis of different regimes might differ in longer period.

\section{INTRODUCTION}

Neovascular age-related macular degeneration (nAMD) is one of the leading causes of severe visual impairment among the elderly both in developed and developing countries. ${ }^{1-4}$ The hallmark of nAMD is choroidal neovascularisation (CNV), which typically leaks and bleeds, then evolves into fibrous metaplasia, permanent loss of photoreceptors and disciform scarring. Most of these cases might result in the irreversible loss of central vision. With the ageing of the population worldwide, the global prevalence of nAMD is estimated to reach 288 million by 2040 , which is a staggering burden and calling for effective and affordable treatment regimens. ${ }^{5}$ Thermal laser photocoagulation and verteporfin photodynamic therapy (PDT) was ever solely used for nAMD. Unfortunately, neither of them could offer any chance for visual improvement. ${ }^{6}$ Later, the advent of antivascular endothelial growth factor (anti-VEGF) revolutionised the treatment of nAMD, which has greatly improved the prognosis of these 
patients by blocking the angiogenesis and inducing the regression of the abnormal blood vessels. ${ }^{8}$

Ranibizumab (Lucentis, Novartis Pharma AG, Basel, Switzerland; and Genentech, South San Francisco, California, USA) was the first anti-VEGF agent approved for the treatment of nAMD. The original studies proved that monthly injections of ranibizumab had substantial effects on visual improvement. ${ }^{9}$ However, this indefinite fixed monthly injection is a substantial burden for both the patients and the healthcare systems. Few ophthalmologists choose this treatment strategy at present. Furthermore, the ceiling efficacy of ranibizumab monotherapy has been reached by monthly injection, and no additional benefit is achieved by increased anti-VEGF dosage. ${ }^{1011}$ Therefore, this raises two core questions: what is the optimum dosage and treatment regimen of ranibizumab? Is there any combined therapy that can improve the efficacy and affordability of ranibizumab? For the first question, pro re nata (PRN) and treat and extend (T\&E) are two widely accepted treatment regimens. Several studies reported they could achieve comparable efficacies with fewer injections and visits. ${ }^{12-15}$ For the second issue, numerous combination approaches have been explored. First of all, the combination of PDT could cause the thrombotic occlusion of $\mathrm{CNV}$, especially for the more mature vessels which may not be sensitive to antiVEGF agents. The simultaneous administration of antiVEGF agents could further suppress the release of VEGF and the formation of neovascularisation after $\mathrm{PDT}^{16}$ Second, as inflammation also plays a crucial role in the development of $\mathrm{CNV}$, it is reasonable to hypothesise the combination of topical non-steroidal anti-inflammatory drugs (NSAIDs) or steroids could produce synergistic or additive effects. ${ }^{17-19}$ Third, some novel agents like tyrosine kinase inhibitors were also evaluated for any extra benefits. $^{20}$

Currently, more than 20 therapeutic regimens of ranibizumab exist for nAMD8. However, no agreement was achieved regarding to the optimum strategy. One reason is that the current randomised controlled trials (RCT) could only achieve a pairwise comparison between these strategies. For example, several RCTs reported that ranibizumab $0.5 \mathrm{mg}$ administered with T\&E regimen was clinically comparable with the monthly regimen in improving visual acuity (VA), while no RCTs had compared the T\&E regimen with $3+\mathrm{PRN}$ regimen or $1+\mathrm{PRN}$ regimen, with or without PDT, NSAIDs or even dexamethasone. ${ }^{12} 1321$ Moreover, some controversies still exist between the same comparisons, some studies suggest that the addition of PDT confers no benefit in terms of VA, while others demonstrate PDT can reduce the retreatment and achieve VA improvement. ${ }^{1822} 23$ Additionally, the previously regular meta-analysis and systemic review did only achieve pairwise comparison between two regimens, which could not provide a comprehensive assessment of the currently regimens of ranibizumab. ${ }^{812} 2425$ Although several network meta-analysis had evaluated the efficacy of ranibizumab treatment for nAMD, the safety and some other anatomic parameters have not been analysed, some special regimens like ranibizumab plus NSAIDs were also not inlcuded. ${ }^{26} 27$

Therefore, we performed this network meta-analysis regarding different therapeutic regimens of ranibizumab, which includes all the available data of RCTs and considers all the strategies and combined therapies. We intend to give a comprehensive efficacy and safety ranking of these regimens, thus providing a reference for the decision-making of ophthalmologists.

\section{METHODS}

\section{Search strategy and selection criteria}

This network meta-analysis was conducted according to PRISMA guidelines. ${ }^{28}$ RCTs were identified through a literature search on PubMed, Embase and the Cochrane Central Register of Controlled Trials from their earliest entries through 1 October 2019. Then, the ClinicalTrials. gov, the WHO International Clinical Trials Registry Platform, company-specific trial registries were screened to ensure that all the trials had been identified. The following keywords or corresponding Medical Subject Headings (Mesh) were used: 'Macular Degeneration', 'Ranibizumab' and 'Randomized Controlled Trial'. The detailed electronic search strategy of PubMed was ${ }^{(}((()($agerelated macular degeneration[Title/Abstract]) OR age related macular degeneration[Title/Abstract]) OR macular degeneration[Title/Abstract]) OR AMD[Title/ Abstract]) OR "Macular Degeneration"(Mesh))) AND ((( ("Randomized Controlled Trial” (Publication Type)) OR Randomized Controlled Trial[Title/Abstract]) OR random*[Title/Abstract]) OR RCT[Title/Abstract])) AND (( ("Ranibizumab"(Mesh)) OR Ranibizumab[Title/ Abstract]) OR Lucentis[Title/Abstract]). The reference lists of the retrieved publication and relevant metaanalysis in this discipline were also manually examined to further identify potentially eligible RCTs, which evaluated different therapeutic regimens of ranibizumab for nAMD. Only human studies published in English were considered. This study was performed in accordance with the PRISMA guideline of network meta-analysis and registered at PROSPERO: International prospective register of systematic reviews (CRD42020156353).

\section{Study selection and data extraction}

The inclusion criteria of our study were: (1) participants: patients with CNV secondary to nAMD; (2) intervention: intravitreal injection of ranibizumab; (3) comparison: different dosage or combined therapy or therapeutic strategy of ranibizumab; (4) outcomes: 1-year follow-up with at least one of the followings: VA; lines of VA improvement; central retinal thickness (CRT); the number of ranibizumab injections; incidence of severe ocular adverse events and incidence of adverse events of cardiocerebral vascular system and (5) methodological criterion: RCTs. 
The exclusion criteria were: (1) studies could not be included in the main closed loop (the study which had no cross-regimen with other included studies); (2) insufficient data to estimate an OR or standardised mean difference (SMD); (3) animal studies or cadaver subjects; and (4) duplicated publications.

After screening the titles and abstracts, obtaining the full text of each article and reviewing them, RCTs that met the inclusion criteria and fail the exclusion criteria were included. Two authors (ZXY and MLH) independently extracted and collated the following data using a standardised protocol: first author, publication year, design, group size, patient age, gender ratio, details of interventions, details of outcomes and follow-up periods. For updated publications with the same cohort of patients of the previous study, the data were extracted synthetically. Discrepancies were evaluated by the kappa test, and agreements were achieved by discussing with the corresponding author (CYX). The corresponding authors of the included articles would be contacted if the essential data were unavailable. The Cochrane risk of bias assessment tool was used to assess the methodological quality and risk of bias. ${ }^{29}$

\section{Outcomes}

The primary efficacy outcome of interest was the letter changes (early treatment diabetic retinopathy study, ETDRS) of best-corrected visual acuity (BCVA) in 1 year and the primary safety outcome of interest was the incidence of severe ocular adverse events, including vitreous haemorrhage, rhegmatogenous retinal detachment, endophthalmitis, retinal tear and retinal pigment epithelium tear. Secondary outcomes were the percentage of patients with a gain of three lines or more of BCVA, changes of CRT, number of retreatments and incidence of adverse events of cardiocerebral vascular system.

\section{Data synthesis and statistical analysis}

The efficacy and safety of the competing therapeutic regimens of ranibizumab were estimated by using SMD for continuous outcomes and OR for dichotomous outcomes. For multiarmed trials, we extracted the continuous and dichotomous data from each arm and conducted the analysis. The data of different dosages and fluences of PDT were combined and regarded as general PDT. This is a contrast-based random-effects analysis, and we conducted this network meta-analysis with indirect and mixed comparisons in Stata V.14.0 (Stata, College Station, Texas, USA) using the mvmeta command, network command and self-programmed Stata routines. ${ }^{30-33}$ Cochran $\mathrm{Q}$ test and the $I^{2}$ statistic were used to assess the heterogeneity. ${ }^{34}$ The global inconsistency test by fitting design-bytreatment in the inconsistency model was used to evaluate the level of heterogeneity between direct and indirect estimates. ${ }^{3536}$ Then, the local inconsistency was evaluated by node-splitting method. ${ }^{35}$ The loop-specific approach which assesses the difference between direct and indirect estimates for a specific comparison in the loop was also applied to check the presence of inconsistency. ${ }^{37}$ If the results of these inconsistency tests were acceptable $(p>0.05)$, the consistency model would be selected to compare all the regimens using direct and indirect data. ${ }^{31}{ }^{32}$ To figure out the possible ranking and obtain the intervention hierarchy of competing regimens in the network meta-analysis, the rankograms, surface under the cumulative ranking (SUCRA) curves and the mean ranks were estimated..$^{38}$ A higher SUCRA means superior efficacy or safety. The comparison-adjusted funnel plot was applied to clarify the publication bias of each outcome. ${ }^{39}$ If heterogeneity or inconsistency was substantial in any outcome $(\mathrm{p}<0.05)$, both sensitivity analysis and subgroup analyses (publication year, sample size and so on) would be conducted to identify the source of the heterogeneity. If the heterogeneity or inconsistency could not be eliminated, the pooled result of this specific outcome would be regarded as invalid.

The quality of evidence contributing to network estimates of each outcome was assessed with the Grading of Recommendations Assessment, Development and Evaluation (GRADE) framework described by Salanti and collegues. ${ }^{40}$ The quality of evidence was graded into four levels: high, moderate, low and very low, which was based on five domains: study limitations, imprecision, indirectness, inconsistency and publication bias.

\section{Role of the funding source}

There was no funding source for this study. The corresponding author had full access to all the data in the study and had final responsibility for the decision to submit for publication.

\section{Patient and public involvement}

Since this is a systematic review and meta-analysis, patients and the public were not involved in the design, or planning of the study.

\section{RESULTS}

\section{Study characteristics}

We identified 1298 citations by the initial search, then 128 potentially eligible articles were retrieved in the full text after reviewing the titles and abstracts. Of these studies, 102 reports were excluded for the following reasons: unrelated interventions or non-RCTs $(n=78)$, comparing one therapeutic regimen of ranibizumab with other antiVEGF agents $(n=11)$, follow-up period shorter than 12 months $(n=4)$ or duplicated publications of the same cohort of patients with longer follow-up $(n=9)$. Finally, 26 studies that met the inclusion criteria were available for inclusion in this analysis ${ }^{9111315-18222341-57}$ (figure 1). The inter-rater agreement was excellent between the investigators regarding eligibility $(\kappa=0.76)$

These studies were published between November 2006 and August 2019. In total, 10821 patietns with nAMD (10821 eyes) were included in our analysis. The mean age of patients ranged from 73 to 83 years, and about 


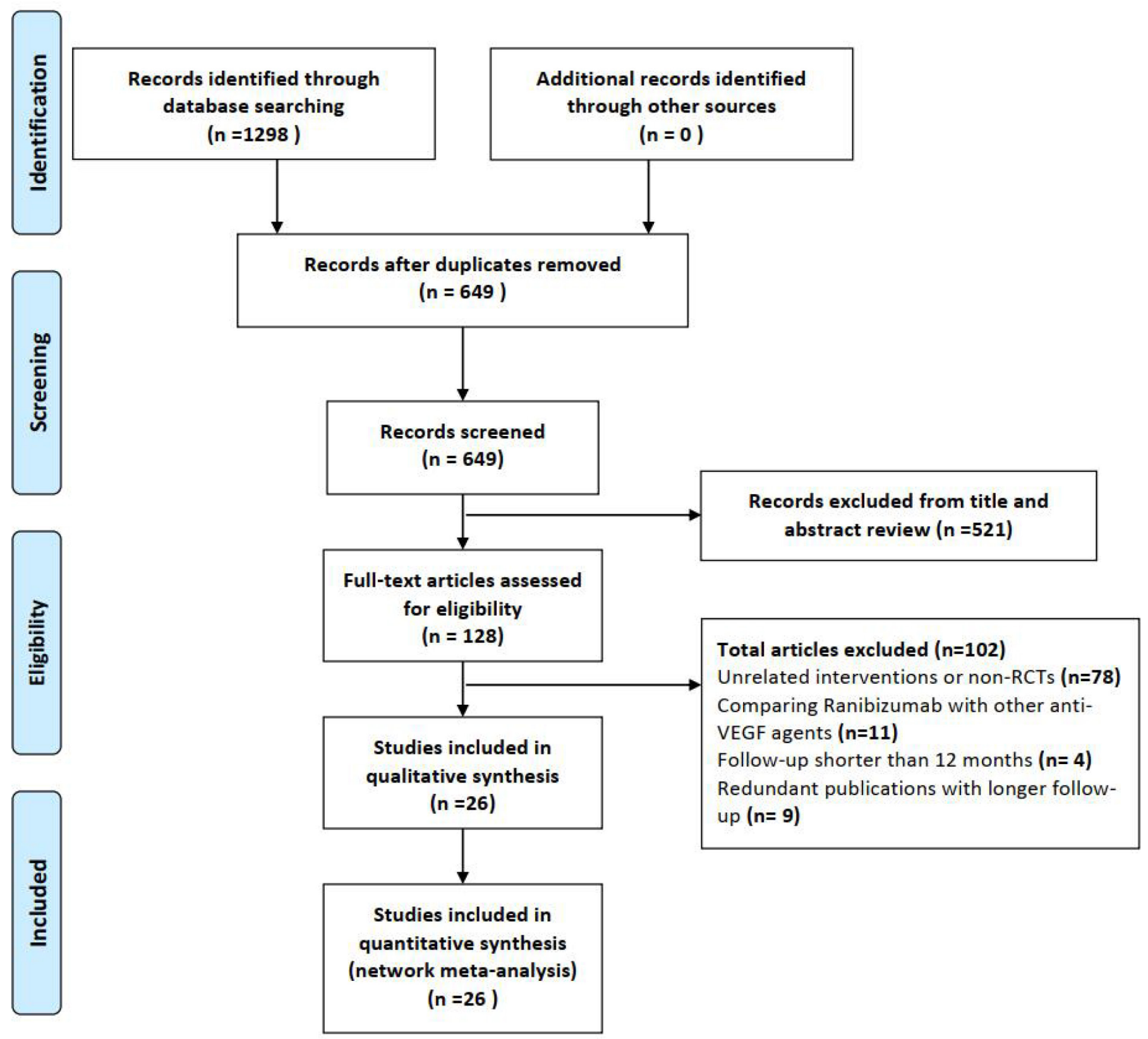

Figure 1 Flowchart depicting the selection of included studies. RCT, randomised controlled trial; VEGF, vascular endothelial growth factor.

$59.4 \%$ of them were women. The main characteristics of these included studies are presented in table 1 . Twenty-two nodes were included in our network meta-analysis, 20 of which were different dosage or combined therapy or therapeutic strategy of ranibizumab, and the other two were PDT and sham treatment (figure 2).

In general, most of these studies (23 of 26) were judged to have an unclear risk of bias (online supplemental files 1 and 2), as only three studies were regarded as low risk in terms of allocation concealment. None of these studies had evidence of a definite high risk in any item. The percentage of studies with low risk of bias in each item was: $34.6 \%$ for randomisation, $11.5 \%$ for allocation concealment, $80.8 \%$ for blinding of participants and personnel, $88.5 \%$ for blinding of outcome assessment and all $100 \%$ for the rest three items.

\section{Primary outcomes}

The network diagrams of all eligible comparisons for the primary outcomes of efficacy and safety are presented in figure 2 and the results of network meta-analysis are shown in figure 3. For all the therapeutic regimens, the efficacy was shown to be greater than the sham group $(p<0.05)$ and safety was similar to the sham group $(p>0.05)$. The mean ranking based on SUCRA curves of the primary efficacy and safety outcomes are shown in table 2 and the detailed results of head-to-head comparisons are provided in table 3. A higher SUCRA means superior efficacy or safety. 


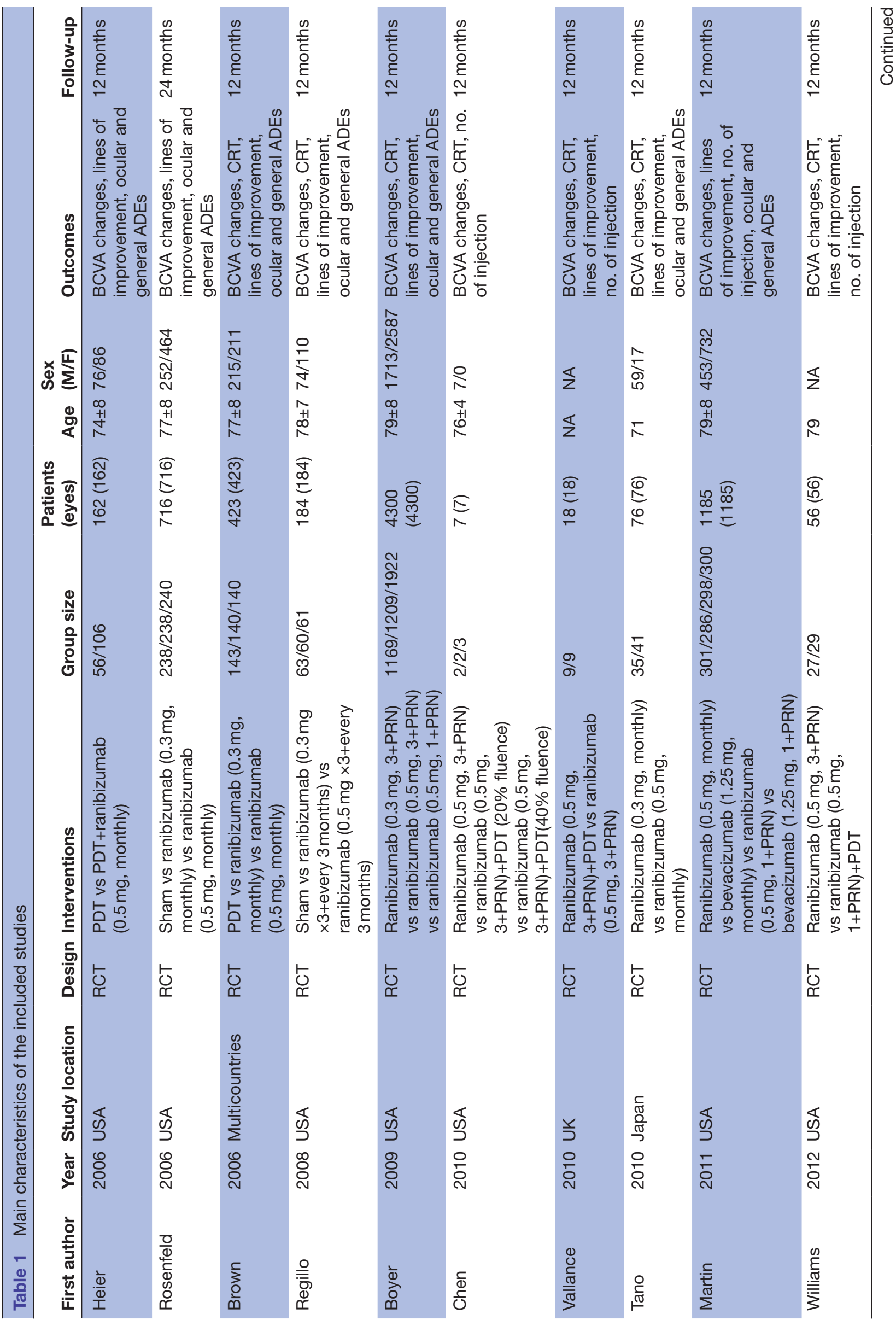




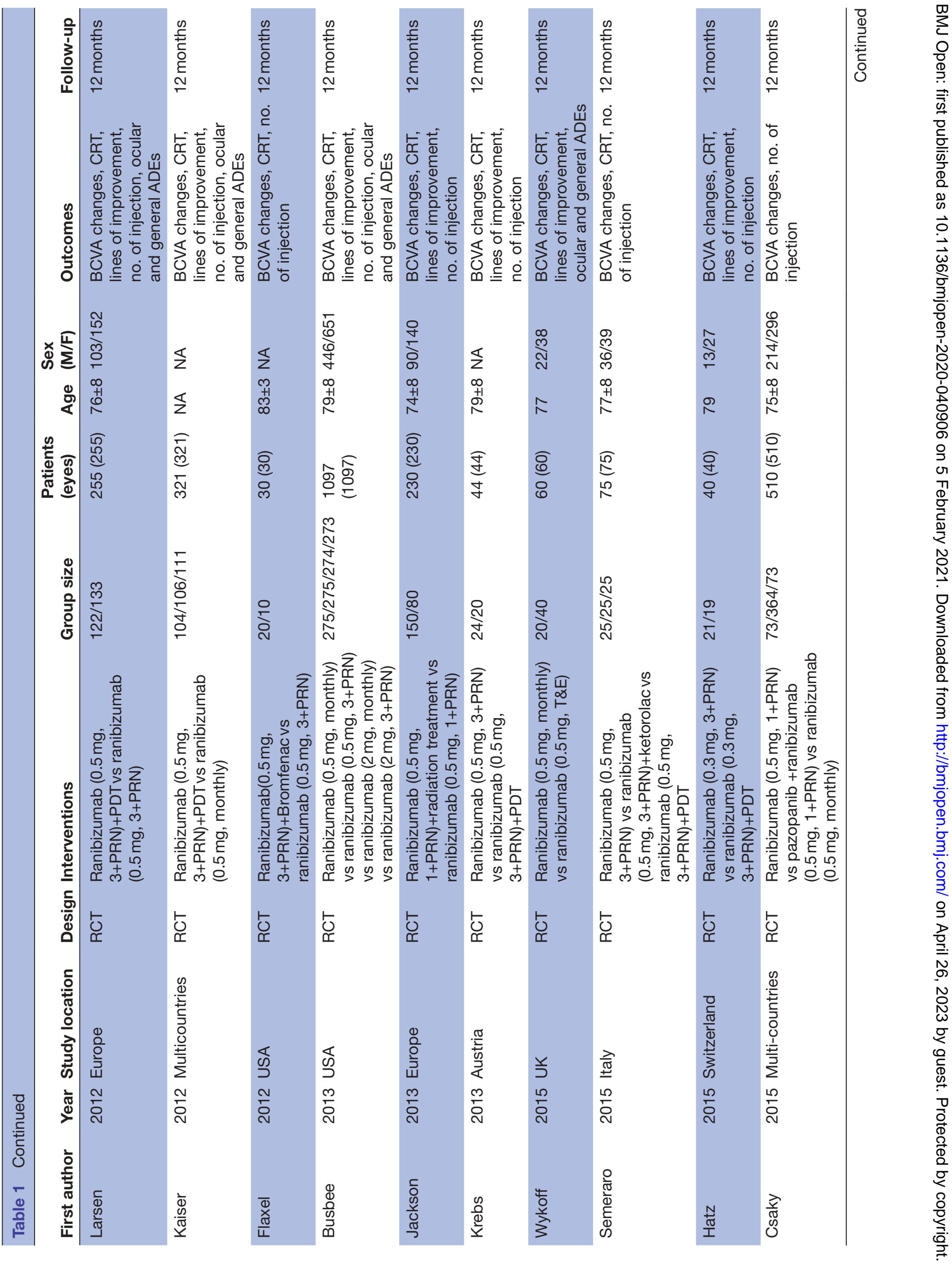




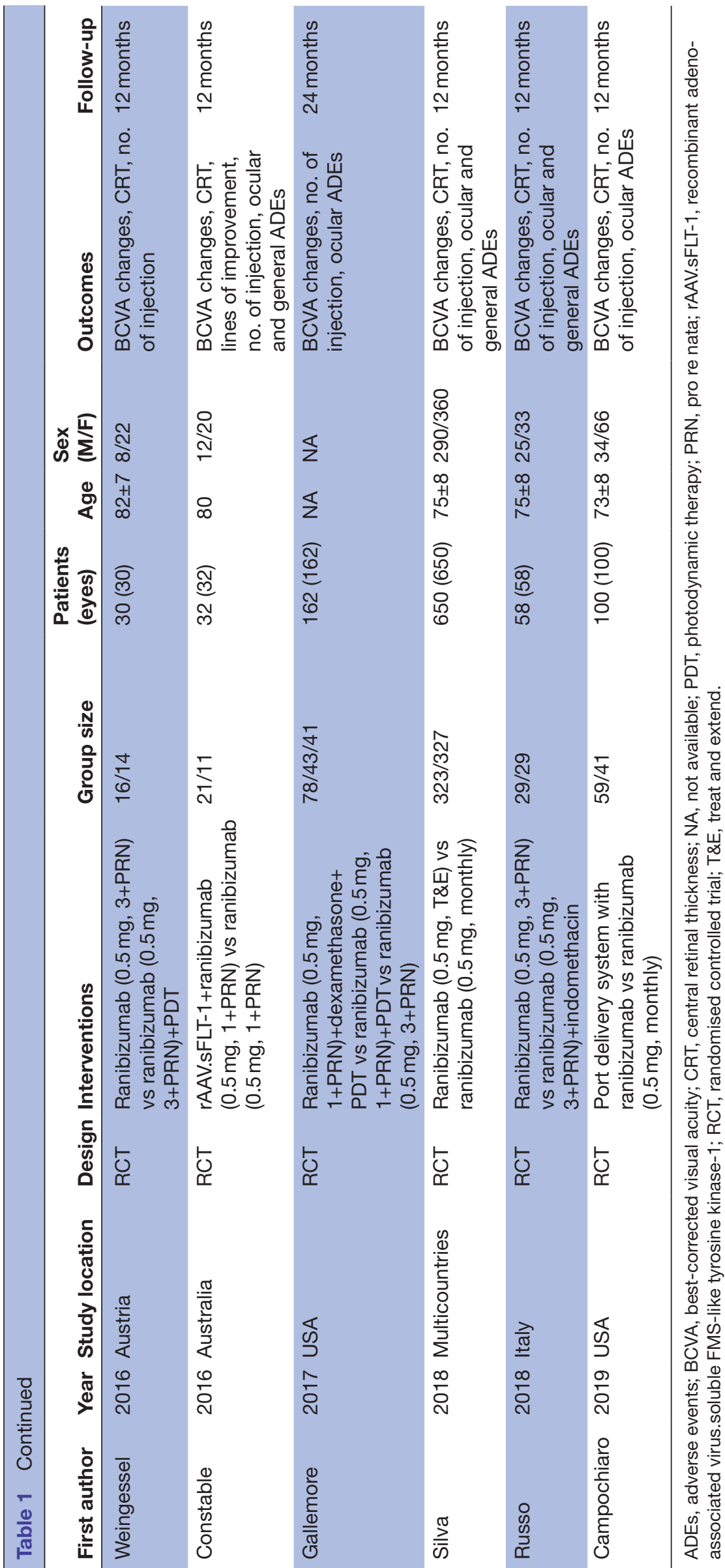


A

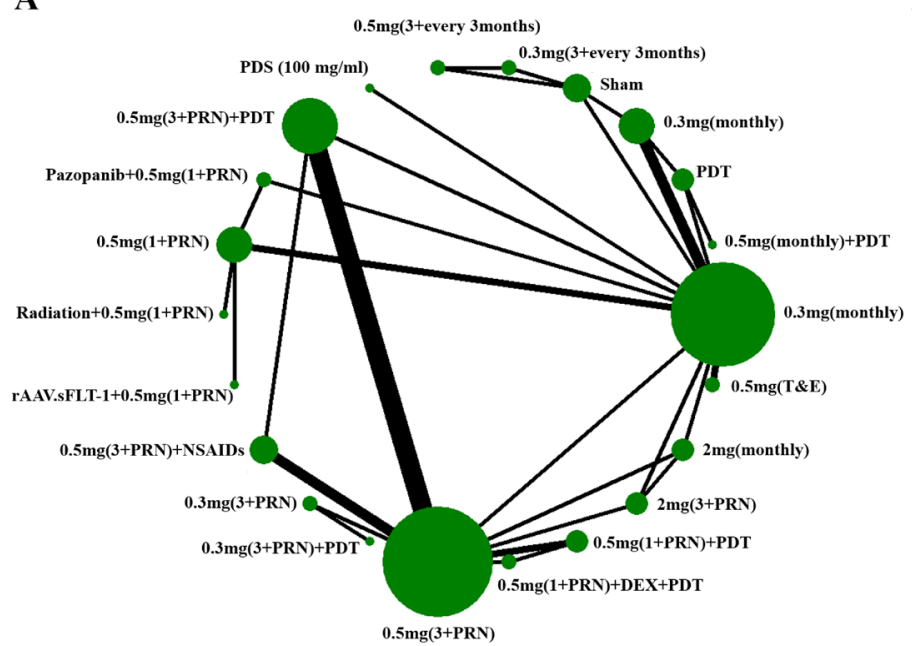

B

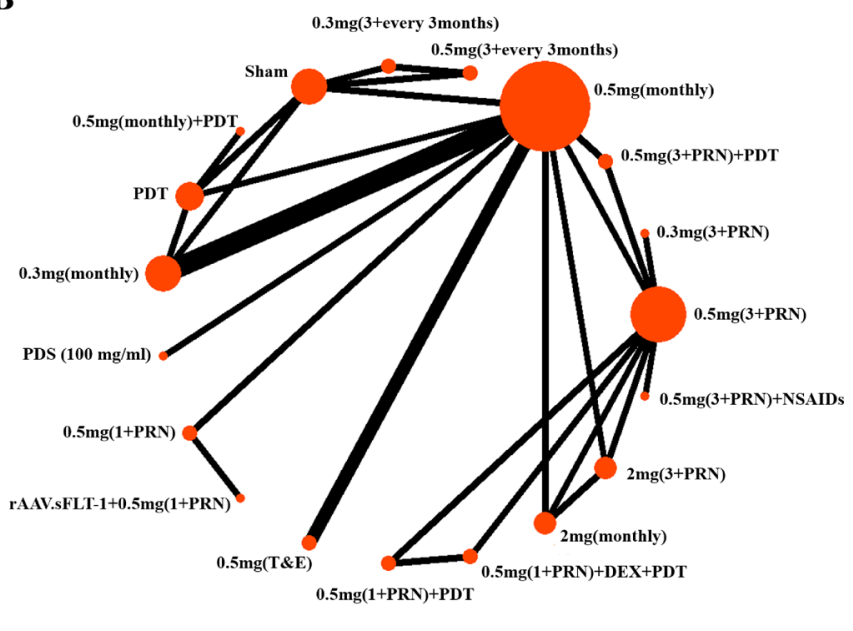

Figure 2 The network diagrams of all eligible comparisons for the primary outcomes of efficacy and safety: (A) letters of bestcorrected visual acuity changes and (B) incidence of severe ocular adverse events. This figure was made by Xinyu Zhao and had got his permission to be published in this article. DEX, dexamethasone; NSAIDs, non-steroidal anti-inflammatory drugs; PDS, port delivery system; PDT, photodynamic therapy; PRN, pro re nata; T\&E, treat and extend.

Twenty-six RCTs involving 6887 patients provided adequate data for the primary efficacy outcome of letters of BCVA changes (ETDRS) at 1 year and the most effective regimen was estimated to be ranibizumab $0.5 \mathrm{mg}$ (T\&E). Compared with the sham group, these 21 therapeutic regimens significantly increased the number of
A

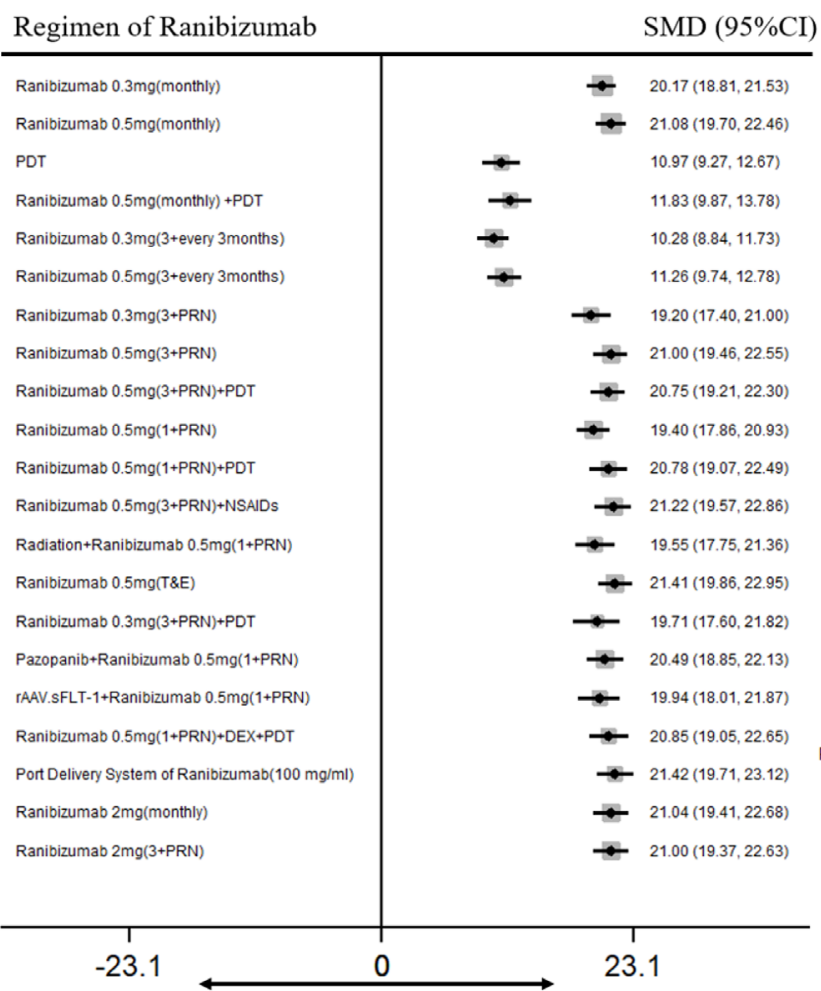

Favours sham treatment $\quad$ Favours active interventions

B

Regimen of Ranibizumab

Ranibizumab $0.3 \mathrm{mg}$ (monthly)
Ranibizumab $0.5 \mathrm{mg}$ (monthly)
PDT
Ranibizumab $0.5 \mathrm{mg}$ (monthly) $+\mathrm{PDT}$
Ranibizumab $0.3 \mathrm{mg}$ (3+every 3 months $)$
Ranibizumab $0.5 \mathrm{mg}(3+$ every 3 months $)$
Ranibizumab $0.3 \mathrm{mg}(3+\mathrm{PRN})$
Ranibizumab $0.5 \mathrm{mg}(3+\mathrm{PRN})$
Ranibizumab $0.5 \mathrm{mg}(3+\mathrm{PRN})+\mathrm{PDT}$
Ranibizumab $0.5 \mathrm{mg}(\mathrm{T} \& \mathrm{EE})$
Ranibizumab $0.5 \mathrm{mg}(1+\mathrm{PRN})$

rAAV.sFLT-1+Ranibizumab $0.5 \mathrm{mg}(1+P R N)$

Ranibizumab $0.5 \mathrm{mg}(1+\mathrm{PRN})+\mathrm{DEX}+\mathrm{PDT}$

Ranibizumab $0.5 \mathrm{mg}(1+\mathrm{PRN})+\mathrm{PDT}$

Ranibizumab $0.5 \mathrm{mg}(3+\mathrm{PRN})+\mathrm{NSAIDs}$

Port Delivery System of Ranibizumab( $100 \mathrm{mg} / \mathrm{ml})$

Ranibizumab 2mg(monthly)

Ranibizumab 2mg(3+PRN)
OR $(95 \% \mathrm{CI})$

$0.85(-0.08,1.79)$

$0.45(-0.56,1.45)$

$0.45(-1.15,2.05)$

$0.02(-1.78,1.83)$

$0.05(-3.89,3.98)$

$0.03(-3.90,3.97)$

$-0.77(-3.17,1.62)$

$-0.54(-2.41,1.32)$

$0.25(-1.50,2.01)$

$1.34(-0.77,3.46)$

$-1.16(-4.36,2.04)$

$-0.02(-3.96,3.91)$

$0.46(-3.13,4.04)$

$1.07(-2.52,4.66)$

$-0.54(-4.91,3.83)$

$2.92(-0.14,5.98)$

$-0.17(-2.12,1.79)$

$-0.86(-3.26,1.54)$

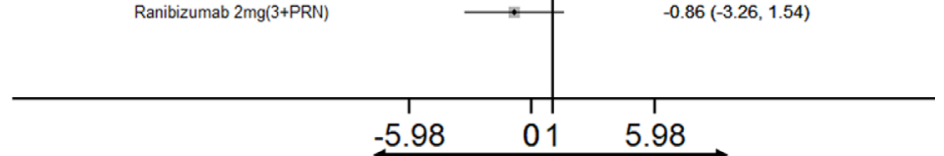

Favours active interventions Favours sham treatment

Figure 3 Network meta-analysis of different regimens of ranibizumab compared with sham treatment for the primary outcomes: (A) letters of best-corrected visual acuity changes and (B) incidence of severe ocular adverse events. DEX, dexamethasone. NSAIDs, non-steroidal anti-inflammatory drugs; PDT, photodynamic therapy; PRN, pro re nata; SMD, standardised mean difference; T\&E, treat and extend. 


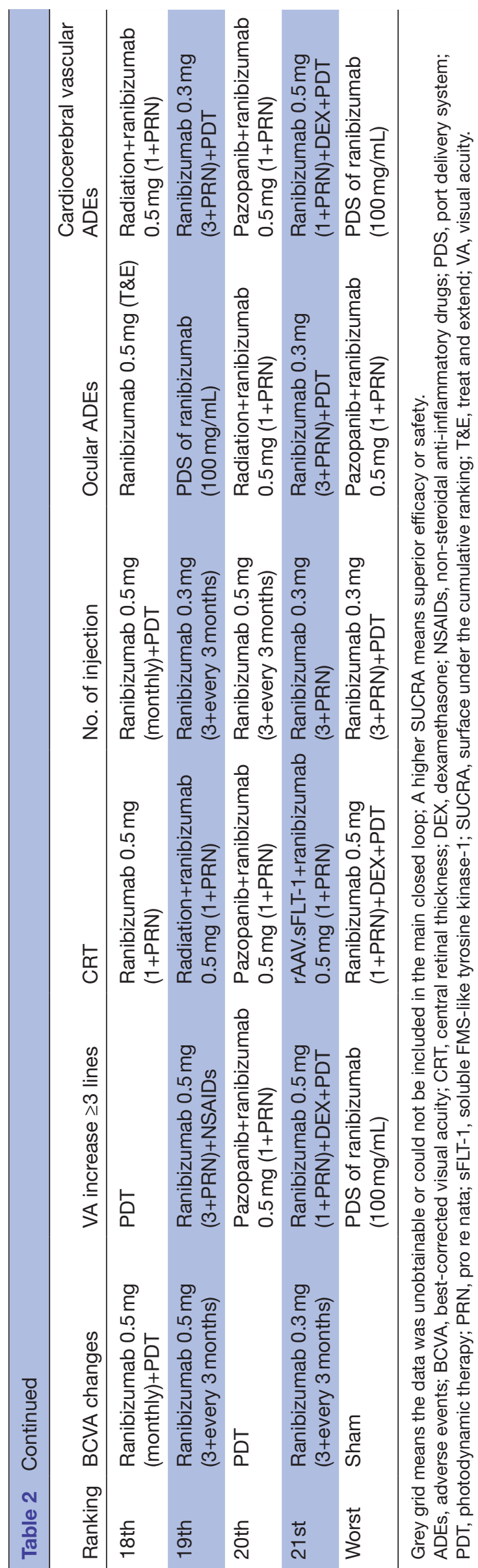

BCVA letters, with SMDs ranging from 10.28 (95\% CI 8.84 to 11.73 , figure 3 ) for ranibizumab $0.3 \mathrm{mg}$ (3+every 3 months) to 21.41 (95\% CI 19.86 to 22.95 ) for ranibizumab $0.5 \mathrm{mg}$ (T\&E).

Sixteen RCTs involving 5845 patients evaluated the incidence of severe ocular adverse events and the network meta-analysis indicated that there was no statistical difference between the 18 therapeutic regimens and the sham treatment. The ORs ranged from $-1.16(95 \% \mathrm{CI}-4.36$ to 2.04, figure 3) for ranibizumab $0.5 \mathrm{mg}(1+\mathrm{PRN})$ to 2.92 (95\% CI -0.14 to 5.98$)$ for port delivery system of ranibizumab $(100 \mathrm{mg} / \mathrm{mL})$.

\section{Secondary outcomes}

The results of the network meta-analysis are shown in figure 4. The corresponding mean ranking based on SUCRA curves is also listed in table 2, a higher SUCRA means superior efficacy or safety. For all the secondary outcomes, the network diagrams and detailed results of head-to-head comparisons are provided in online supplemental files 3-7.

Seventeen RCTs involving 5263 patients reported the percentage of patients with a gain of three lines or more of BCVA (ETDRS) and ranibizumab $0.5 \mathrm{mg}$ (T\&E) was estimated to have the highest SUCRA ranking. Compared with the sham group, seven therapeutic regimens achieved significantly higher ORs, ranging from 1.77 (95\% CI 1.05 to 2.49$)$ for ranibizumab $0.5 \mathrm{mg}(1+\mathrm{PRN})$ to 2.83 (95\% CI 1.27 to 4.38) for ranibizumab $0.5 \mathrm{mg}$ (T\&E), while no statistical difference existed for the rest regimens (figure 4).

Eighteen RCTs involving 4292 patients reported the changes of CRT and the most effective regimen was estimated to be ranibizumab $0.5 \mathrm{mg}(3+\mathrm{PRN})+\mathrm{NSAIDs}$. Compared with PDT, all the other 12 therapeutic regimens achieved significantly better reduction of CRT, ranging from $-2.04(95 \% \mathrm{CI}-3.73$ to -0.35$)$ for ranibizumab $0.3 \mathrm{mg} \quad(3+\mathrm{PRN})+\mathrm{PDT}$ to $-3.45 \quad(95 \%$ CI -4.63 to -2.28$)$ for ranibizumab $0.5 \mathrm{mg}(3+\mathrm{PRN})+\mathrm{NSAIDs}$ (figure 4).

Eighteen RCTs involving 4274 patients reported the number of retreatments and the port delivery system of ranibizumab $(100 \mathrm{mg} / \mathrm{mL})$ was shown to require the least number of injections (figure 4). Compared with ranibizumab $0.5 \mathrm{mg}$ (monthly), 11 therapeutic regimens required significantly less number of retreatments, while no statistical difference was detected for ranibizumab $0.5 \mathrm{mg}$ (T\&E) (SMD $=-0.94,95 \% \mathrm{CI}-2.26$ to 0.39$)$ and ranibizumab $2 \mathrm{mg}$ (monthly) ( $\mathrm{SMD}=-0.08,95 \% \mathrm{CI}-1.33$ to 1.18$)$.

Fourteen RCTs involving 5583 patients evaluated the incidence of adverse events of cardiocerebral vascular system (figure 4). The network meta-analysis showed that PDT had a lower risk ( $\mathrm{OR}=-0.34 ; 95 \% \mathrm{C}:-1.47$ to 0.79$)$, while no significant difference existed between the other 14 therapeutic regimens and the sham treatment. The ORs ranged from -0.68 (95\% CI -2.41 to 1.06 , figure 3 ) for ranibizumab $0.3 \mathrm{mg}$ (3+every3 months) to 1.31 (95\% 


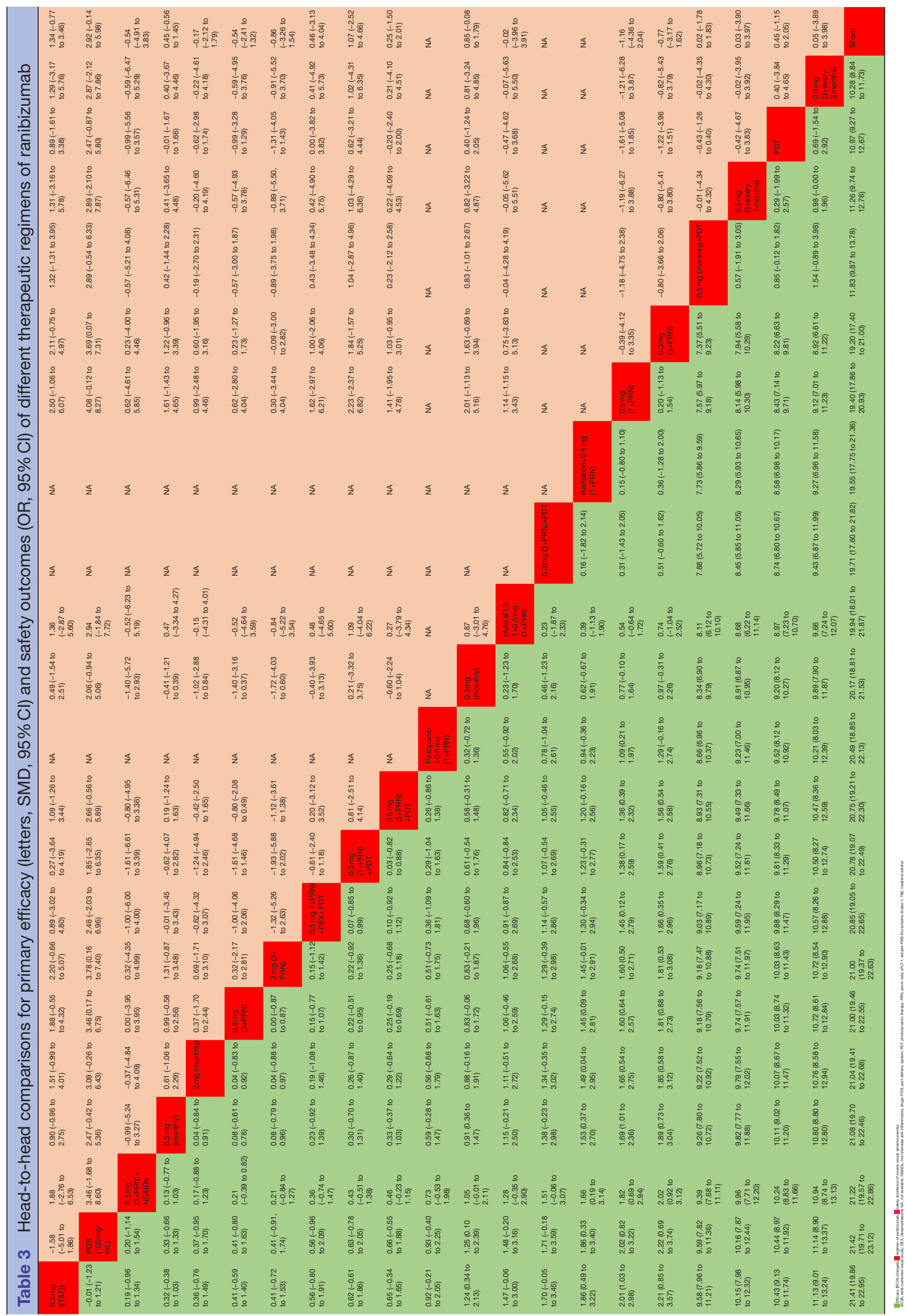


A

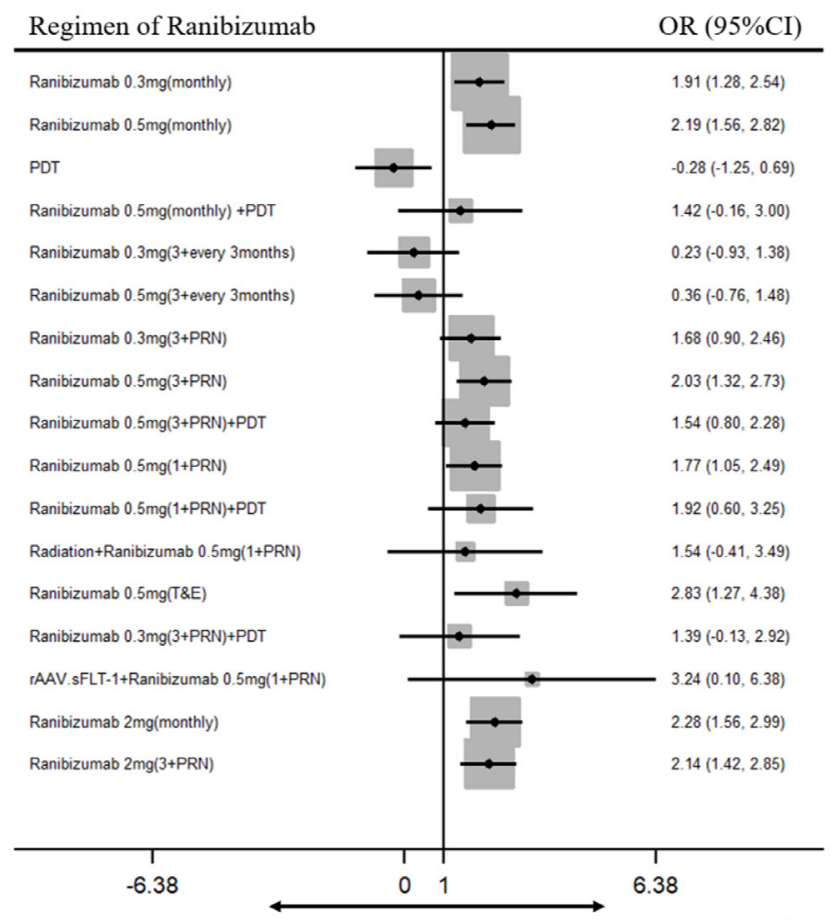

Favours sham treatment
Favours active interventions
B

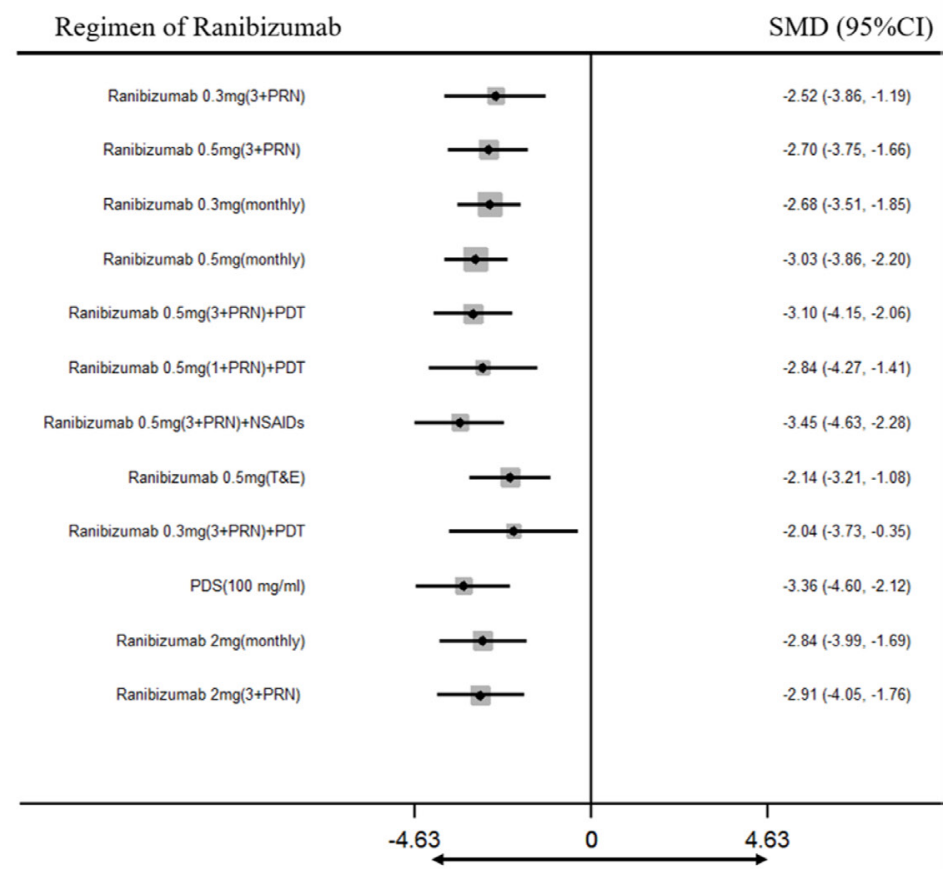

Favours active interventions

Favours PDT

D

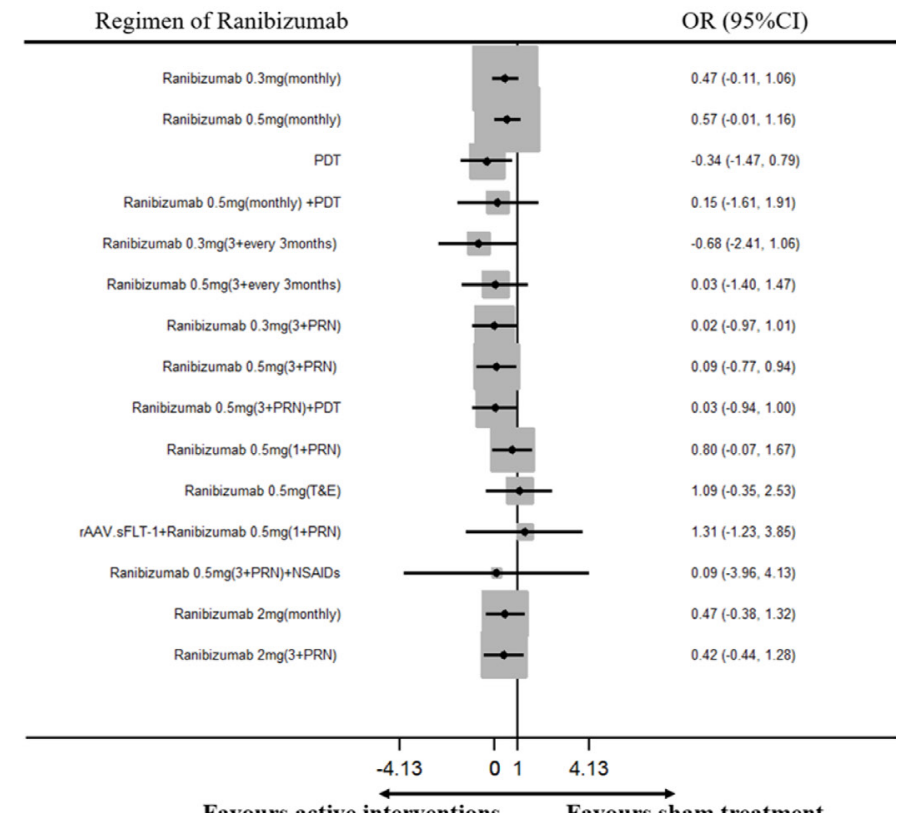

Favours active interventions $\quad$ Favours sham treatment

Figure 4 Network meta-analysis of different regimens of ranibizumab for the secondary outcomes: (A) gain of three lines or more of best-corrected visual acuity, compared with sham treatment; (B) changes of central retinal thickness, compared with PDT; (C) number of retreatments, compared with ranibizumab $0.5 \mathrm{mg}$ (monthly) and (D) incidence of adverse events of cardiocerebral vascular system, compared with sham treatment. DEX, dexamethasone; NSAIDs, non-steroidal anti-inflammatory drugs; PDS, port delivery system; PDT, photodynamic therapy; PRN, pro re nata; SMD, standardised mean difference; T\&E, treat and extend. 
CI -1.23 to 3.85$)$ for rAAV.sFLT-1+ranibizumab $0.5 \mathrm{mg}$ $(1+\mathrm{PRN})$. The safety of each regimen was compared with the sham group, so the lower limit could be negative.

\section{Inconsistency or heterogeneity}

Global inconsistency, local inconsistency or heterogeneity was not significant between evidence derived from direct and indirect comparisons in both of the primary and secondary outcomes $(\mathrm{p}>0.05)$. The corresponding comparison-adjusted funnel plots also showed no evidence of asymmetry $(\mathrm{p}>0.05)$.

\section{DISCUSSION}

This study is based on 26 RCTs, which included 10821 patients with nAMD randomly assigned to 21 different therapeutic regimens of ranibizumab or sham treatment. To the best of knowledge, this study is the largest and most comprehensive network meta-analysis in evaluating the efficacy and safety of different regimens of ranibizumab for nAMD. More importantly, it assessed safety outcomes and other secondary outcomes such as CRT reduction for the first time in a way of network meta-analysis. This large evidence base, obtained through exhaustive data screening, extraction and analysis, might provide solid guidance for both ophthalmologists and patients. Our findings can be summarised as follows: ranibizumab $0.5 \mathrm{mg}$ (T\&E) is the most effective strategy for the treatment of nAMD, with no more risk of severe ocular adverse events or cardiocerebral vascular complications compared with sham treatment (weak). However, this strategy could not significantly reduce the number of retreatment compared with monthly injection (weak). Ranibizumab $0.5 \mathrm{mg}(3+\mathrm{PRN})+\mathrm{NSAIDs}$ shows the best effect in reducing CRT (weak) and the port delivery system of ranibizumab $(100 \mathrm{mg} / \mathrm{mL})$ is the most effective method in reducing the number of retreatment, with acceptable risks of adverse events (weak). The quality of evidence was assessed by the GRADE framework (online supplemental files 8-13).

For the efficacy of ranibizumab for nAMD, previous studies mainly held the opinion that the highest benefit could be achieved in patients who were monitored and treated monthly. ${ }^{16} 5758$ However, this strategy places tremendous economic pressure both on the patients and the healthcare system. In response to all these realworld burdens, alternate strategies like PRN and T\&E were devised. Compared with monthly injection and PRN, T\&E was aimed to reduce both the number of injections and visits. The results of our network meta-analysis indicated that the T\&E protocol achieved the highest SUCRA ranking in terms of the increasing number of BCVA letters and the percentage of patients with a gain of three lines or more of BCVA (weak). Although the pairwise comparisons between T\&E, PRN and monthly injection showed no significant difference $(\mathrm{p}<0.05)$, the mean ranking based on SUCRA curves could still prove that the
T\&E protocol is an effective alternative with higher costefficiency (weak).

The combinatorial efficacy of PDT seems to depend on the original protocols of ranibizumab and all of them get a much lower SUCRA ranking compared with ranibizumab $0.5 \mathrm{mg}$ (T\&E) or a monthly injection. For the outcome of BCVA changes, the combination of PDT was negative on ranibizumab $0.5 \mathrm{mg}$ (monthly), positive on $0.5 \mathrm{mg}(1+\mathrm{PRN})$, while no significant difference on $0.5 \mathrm{mg}$ $(3+\mathrm{PRN})$ (weak). Therefore, the combination of PDT might not be that necessary as ranibizumab monotherapy could already achieve satisfactory outcomes. Besides, radiation therapy also showed no combinatorial benefit.

Some novel agents such as tyrosine kinase inhibitor (topical pazopanib and rAAV.sFLT-1) and port delivery system with ranibizumab $(100 \mathrm{mg} / \mathrm{mL})$ did not gain extra benefits in terms of visual outcomes compared with ranibizumab $0.5 \mathrm{mg}(\mathrm{T} \& \mathrm{E})$, while they achieved a higher SUCRA ranking in reducing the number of retreatments (weak). For the port delivery system, only the dosage of $100 \mathrm{mg} / \mathrm{mL}$ was included in our analysis as the original study showed it had better efficacy and safety compared with other dosages (weak). ${ }^{57}$

Currently, it is well known that VEGF is not the only causative factor of CNV, free radicals, oxidised lipoproteins and the subsequent inflammation all play crucial roles in the development of $\mathrm{CNV} .^{17}$ The results of our network meta-analysis demonstrated that ranibizumab $0.5 \mathrm{mg}(3+\mathrm{PRN})+\mathrm{NSAIDs}$ got the highest SUCRA ranking in CRT reduction, its efficacy was better than ranibizumab $0.5 \mathrm{mg}(3+\mathrm{PRN})$ and even ranibizumab $0.5 \mathrm{mg}$ (TREX) (weak). In the meantime, ranibizumab $0.5 \mathrm{mg}$ $(3+\mathrm{PRN})+$ NSAIDsalso got the third SUCRA ranking in terms of BCVA improvement (weak). All these demonstrate that the administration of topical NSAIDs could achieve additional efficacy in the treatment of nAMD. Additionally, the combination of NSAIDs or dexamethasone could achieve a higher SUCRA ranking of visual outcome than the original protocol (weak).

Although all these therapeutic regimens did not significantly increase the risk of severe ocular or cardiocerebral vascular adverse events compared with sham treatment, we should notice that the SUCRA ranking of Ranibizumab $0.5 \mathrm{mg}$ (T\&E) was relatively low (weak). We speculated the longer follow-up intervals of T\&E protocol might potentially fail to achieve timely intervention for the progress of ocular lesions. As the T\&E protocol could not significantly reduce the number of retreatment compared with monthly injection, the patients were exposed to the same level of ocular injections and VEGF agents in the circulation, which might lead to the same risk of cardiocerebral vascular complications.

To our knowledge, this study is the largest and most comprehensive network meta-analysis in evaluating the efficacy and safety of different regimens of ranibizumab for nAMD. More importantly, it assessed safety outcomes and other secondary outcomes such as CRT reduction for the first time in a way of network meta-analysis. It gives a 
comprehensive efficacy and safety ranking of these regimens, which provides a solid reference for the decisionmaking of ophthalmologists. However, some limitations still exist: (1) only RCTs published in English were considered; (2) as no significant inconsistency or heterogeneity was detected in any outcome, we did not perform sensitivity analysis or subgroup analysis; (3) we could not achieve the full ranking of some outcomes because some data were unobtainable or the studies could not be included in the main closed loop; (4) the follow-up period was limited to 12 months, the results might differ in the longer period and (5) for a network meta-analysis, the studies or regimens which had no cross-regimen with other included studies had to be excluded, as they could not form a closed loop with other regimens, and the data synthesis and statistical analysis could not be conducted.

Based on the results of our network meta-analysis, we summarised that ranibizumab $0.5 \mathrm{mg}$ (T\&E) was the most effective regimen in improving the visual outcome with a less economic burden. The administration of topical NSAIDs could achieve additional efficacy in CRT reduction and visual improvement. Both these two interventions had acceptable risks of adverse events. Therefore, it is reasonable to suggest that further study should be performed to evaluate whether ranibizumab $0.5 \mathrm{mg}$ (T\&E)+NSAIDs could achieve better efficacy than current regimens.

The EVEREST II study had updated the 24-month outcomes of ranibizumab and PDT combination therapy. ${ }^{59}$ Our study did not include it as we set the follow-up period at 12 months.

Acknowledgements We thank Andy Chen from Department of Biomedical Engineering, Indiana University-Purdue University Indianapolis, for his editorial assistance.

Contributors $\mathrm{XZ}$ carried out the entire procedure including the literature search, data extraction, performed the statistical analysis, drafted and revised the manuscript. LM contributed to the revision of the manuscript. YC conceived of the study, coordinated and participated in the entire process of drafting and revising the manuscript.

Funding The authors have not declared a specific grant for this research from any funding agency in the public, commercial or not-for-profit sectors.

Competing interests All authors have contributed significantly and are in agreement with the content of the manuscript. No author has any relevant financial relationship to disclose.

Patient consent for publication Not required.

Ethical approval and informed consent Since this is a systematic review, ethical approval and informed consent are not required.

Provenance and peer review Not commissioned; externally peer reviewed.

Data availability statement Data are available in a public, open access repository. All data relevant to the study are included in the article or uploaded as supplementary information. All data relevant to the study are included in the article or uploaded as online supplemental information. No unpublished data are available. All included articles could be downloaded from the website https://pubmed.ncbi. nlm.nih.gov/.

Supplemental material This content has been supplied by the author(s). It has not been vetted by BMJ Publishing Group Limited (BMJ) and may not have been peer-reviewed. Any opinions or recommendations discussed are solely those of the author(s) and are not endorsed by BMJ. BMJ disclaims all liability and responsibility arising from any reliance placed on the content. Where the content includes any translated material, BMJ does not warrant the accuracy and reliability of the translations (including but not limited to local regulations, clinical guidelines, terminology, drug names and drug dosages), and is not responsible for any error and/or omissions arising from translation and adaptation or otherwise.

Open access This is an open access article distributed in accordance with the Creative Commons Attribution Non Commercial (CC BY-NC 4.0) license, which permits others to distribute, remix, adapt, build upon this work non-commercially, and license their derivative works on different terms, provided the original work is properly cited, appropriate credit is given, any changes made indicated, and the use is non-commercial. See: http://creativecommons.org/licenses/by-nc/4.0/.

ORCID iD

Youxin Chen http://orcid.org/0000-0002-7231-5058

\section{REFERENCES}

1 Bourne RRA, Jonas JB, Flaxman SR, et al. Prevalence and causes of vision loss in high-income countries and in eastern and central Europe: 1990-2010. Br J Ophthalmol 2014;98:629-38.

2 Grunwald JE, Pistilli M, Daniel E, et al. Incidence and growth of geographic atrophy during 5 years of comparison of agerelated macular degeneration treatments trials. Ophthalmology 2017:124:97-104.

3 Zhao X-Y, Xia S, Luo M-Y, et al. The occurrence, characteristics, management, and prognosis of retinal pigment epithelium tears in patients with polypoidal choroidal vasculopathy: a retrospective study of 397 patients. Retina 2020;40:477-89.

4 Kawasaki R, Yasuda M, Song SJ, et al. The prevalence of age-related macular degeneration in Asians: a systematic review and metaanalysis. Ophthalmology 2010;117:921-7.

5 Wong WL, Su X, Li X, et al. Global prevalence of age-related macular degeneration and disease burden projection for 2020 and 2040: a systematic review and meta-analysis. Lancet Glob Health 2014;2:e106-16.

6 Lazic R, Gabric N. Verteporfin therapy and intravitreal bevacizumab combined and alone in choroidal neovascularization due to agerelated macular degeneration. Ophthalmology 2007;114:1179-85.

7 Virgili G, Bini A. Laser photocoagulation for neovascular agerelated macular degeneration. Cochrane Database Syst Rev 2007;3:CD004763.

8 Solomon SD, Lindsley K, Vedula SS, et al. Anti-Vascular endothelial growth factor for neovascular age-related macular degeneration. Cochrane Database Syst Rev 2019;3:CD005139.

9 Rosenfeld PJ, Brown DM, Heier JS, et al. Ranibizumab for neovascular age-related macular degeneration. $N$ Engl J Med 2006;355:1419-31.

10 Heier JS, Brown DM, Chong V, et al. Intravitreal aflibercept (VEGF trap-eye) in wet age-related macular degeneration. Ophthalmology 2012;119:2537-48.

11 Busbee BG, Ho AC, Brown DM, et al. Twelve-month efficacy and safety of $0.5 \mathrm{Mg}$ or $2.0 \mathrm{Mg}$ ranibizumab in patients with subfoveal neovascular age-related macular degeneration. Ophthalmology 2013;120:1046-56.

12 Okada M, Kandasamy R, Chong EW, et al. The Treat-and-Extend injection regimen versus alternate dosing strategies in age-related macular degeneration: a systematic review and meta-analysis. $\mathrm{Am} \mathrm{J}$ Ophthalmol 2018;192:184-97.

13 Silva R, Berta A, Larsen M, et al. Treat-and-extend versus monthly regimen in neovascular age-related macular degeneration: results with ranibizumab from the trend study. Ophthalmology 2018;125:57-65.

14 Ho AC, Busbee BG, Regillo CD, et al. Twenty-four-month efficacy and safety of $0.5 \mathrm{Mg}$ or $2.0 \mathrm{Mg}$ ranibizumab in patients with subfoveal neovascular age-related macular degeneration. Ophthalmology 2014;121:2181-92.

15 Wykoff CC, Croft DE, Brown DM, et al. Prospective trial of treatand-extend versus monthly dosing for neovascular age-related macular degeneration: TREX-AMD 1-year results. Ophthalmology 2015;122:2514-22.

16 Brown DM, Kaiser PK, Michels M, et al. Ranibizumab versus verteporfin for neovascular age-related macular degeneration. $N$ Engl J Med 2006;355:1432-44.

17 Semeraro F, Russo A, Delcassi L, et al. Treatment of exudative age-related macular degeneration with ranibizumab combined with ketorolac eyedrops or photodynamic therapy. Retina 2015;35:1547-54.

18 Gallemore RP, Wallsh J, Hudson HL, et al. Combination verteporfin photodynamic therapy ranibizumab-dexamethasone in choroidal 
neovascularization due to age-related macular degeneration: results of a phase II randomized trial. Clin Ophthalmol 2017;11:223-31.

19 Kuppermann BD, Goldstein M, Maturi RK, et al. Dexamethasone intravitreal implant as adjunctive therapy to ranibizumab in neovascular age-related macular degeneration: a multicenter randomized controlled trial. Ophthalmologica 2015;234:40-54.

20 Rakoczy EP, Magno AL, Lai C-M, et al. Three-Year follow-up of phase 1 and $2 \mathrm{~A}$ rAAV.sFLT-1 subretinal gene therapy trials for exudative age-related macular degeneration. Am J Ophthalmol 2019:204:113-23.

21 Wykoff CC, Ou WC, Croft DE, et al. Neovascular age-related macular degeneration management in the third year: final results from the TREX-AMD randomised trial. Br J Ophthalmol 2018;102:460-4.

22 Weingessel B, Mihaltz K, Vécsei-Marlovits PV. Predictors of 1-year visual outcome in OCT analysis comparing ranibizumab monotherapy versus combination therapy with PDT in exsudative age-related macular degeneration. Wien Klin Wochenschr 2016;128:560-5.

23 Krebs I, Vécsei Marlovits V, Bodenstorfer J, et al. Comparison of ranibizumab monotherapy versus combination of ranibizumab with photodynamic therapy with neovascular age-related macular degeneration. Acta Ophthalmol 2013;91:e178-83.

24 Gao Y, Yu T, Zhang Y, et al. Anti-Vegf monotherapy versus photodynamic therapy and anti-VEGF combination treatment for neovascular age-related macular degeneration: a meta-analysis. Invest Ophthalmol Vis Sci 2018;59:4307-17.

25 Nguyen CL, Oh LJ, Wong E, et al. Anti-Vascular endothelial growth factor for neovascular age-related macular degeneration: a meta-analysis of randomized controlled trials. BMC Ophthalmo 2018;18:130

26 Ohji M, Lanzetta P, Korobelnik J-F, et al. Efficacy and treatment burden of intravitreal aflibercept versus intravitrea ranibizumab treat-and-extend regimens at 2 years: network meta-analysis incorporating individual patient data metaregression and matching-adjusted indirect comparison. Adv Ther 2020;37:2184-98

27 Danyliv A, Glanville J, McCool R, et al. The clinical effectiveness of ranibizumab treat and extend regimen in $\mathrm{nAMD}$ : systematic review and network meta-analysis. Adv Ther 2017;34:611-9.

28 Hutton B, Salanti G, Caldwell DM, et al. The PRISMA extension statement for reporting of systematic reviews incorporating network meta-analyses of health care interventions: checklist and explanations. Ann Intern Med 2015;162:777-84.

29 Higgins JPT, Altman DG, Gøtzsche PC, et al. The Cochrane collaboration's tool for assessing risk of bias in randomised trials. BMJ 2011;343:d5928.

30 White IR, Barrett JK, Jackson D, et al. Consistency and inconsistency in network meta-analysis: model estimation using multivariate metaregression. Res Synth Methods 2012;3:111-25.

31 Caldwell DM, Ades AE, Higgins JPT. Simultaneous comparison of multiple treatments: combining direct and indirect evidence. BMJ 2005;331:897-900.

$32 \mathrm{Lu} \mathrm{G}$, Ades AE. Combination of direct and indirect evidence in mixed treatment comparisons. Stat Med 2004;23:3105-24.

33 Cipriani A, Higgins JPT, Geddes JR, et al. Conceptual and technical challenges in network meta-analysis. Ann Intern Med 2013;159:130-7

34 Higgins JPT, Thompson SG, Deeks JJ, et al. Measuring inconsistency in meta-analyses. BMJ 2003;327:557-60.

35 Dias S, Welton NJ, Caldwell DM, et al. Checking consistency in mixed treatment comparison meta-analysis. Stat Med 2010;29:932-44.

36 Higgins JPT, Jackson D, Barrett JK, et al. Consistency and inconsistency in network meta-analysis: concepts and models for multi-arm studies. Res Synth Methods 2012;3:98-110.

37 Bucher HC, Guyatt GH, Griffith LE, et al. The results of direct and indirect treatment comparisons in meta-analysis of randomized controlled trials. J Clin Epidemiol 1997;50:683-91.

38 Salanti G, Ades AE, loannidis JPA. Graphical methods and numerical summaries for presenting results from multiple-treatment metaanalysis: an overview and tutorial. J Clin Epidemiol 2011;64:163-71.

39 Chaimani A, Higgins JPT, Mavridis D, et al. Graphical tools for network meta-analysis in STATA. PLoS One 2013;8:e76654.

40 Salanti G, Del Giovane C, Chaimani A, et al. Evaluating the quality of evidence from a network meta-analysis. PLoS One 2014;9:e99682.
41 Heier JS, Boyer DS, Ciulla TA, et al. Ranibizumab combined with verteporfin photodynamic therapy in neovascular age-related macular degeneration: year 1 results of the focus study. Arch Ophthalmol 2006;124:1532-42.

42 Regillo CD, Brown DM, Abraham P, et al. Randomized, doublemasked, sham-controlled trial of ranibizumab for neovascular agerelated macular degeneration: PIER study year 1. Am J Ophthalmol 2008;145:239-48.

43 Boyer DS, Heier JS, Brown DM, et al. A phase IIIB study to evaluate the safety of ranibizumab in subjects with neovascular age-related macular degeneration. Ophthalmology 2009;116:1731-9.

44 Chen E, Brown DM, Wong TP, et al. Lucentis using Visudyne study: determining the threshold-dose fluence of verteporfin photodynamic therapy combined with intravitreal ranibizumab for exudative macular degeneration. Clin Ophthalmol 2010;4:1073-9.

45 Vallance $\mathrm{JH}$, Johnson $\mathrm{B}$, Majid MA, et al. A randomised prospective double-masked exploratory study comparing combination photodynamic treatment and intravitreal ranibizumab vs intravitreal ranibizumab monotherapy in the treatment of neovascular agerelated macular degeneration. Eye 2010;24:1561-7.

46 Tano Y, Ohji M, EXTEND-I Study Group. EXTEND-I: safety and efficacy of ranibizumab in Japanese patients with subfoveal choroidal neovascularization secondary to age-related macular degeneration. Acta Ophthalmol 2010;88:309-16.

47 CATT Research Group, Martin DF, Maguire MG, et al. Ranibizumab and bevacizumab for neovascular age-related macular degeneration. N Engl J Med 2011;364:1897-908.

48 Williams PD, Callanan D, Solley W, et al. A prospective pilot study comparing combined intravitreal ranibizumab and half-fluence photodynamic therapy with ranibizumab monotherapy in the treatment of neovascular age-related macular degeneration. Clin Ophthalmol 2012;6:1519-25.

49 Larsen M, Schmidt-Erfurth U, Lanzetta P, et al. Verteporfin plus ranibizumab for choroidal neovascularization in age-related macular degeneration: twelve-month Mont blanc study results. Ophthalmology 2012;119:992-1000.

50 Kaiser PK, Boyer DS, Cruess AF, et al. Verteporfin plus ranibizumab for choroidal neovascularization in age-related macular degeneration: twelve-month results of the DENALI study. Ophthalmology 2012;119:1001-10.

51 Flaxel C, Schain MB, Hamon SC, et al. Prospective randomized controlled trial of combination ranibizumab (Lucentis) and bromfenac (Xibrom) for neovascular age-related macular degeneration: a pilot study. Retina 2012;32:417-23.

52 Jackson TL, Chakravarthy U, Kaiser PK, et al. Stereotactic radiotherapy for neovascular age-related macular degeneration: 52-week safety and efficacy results of the INTREPID study. Ophthalmology 2013;120:1893-900.

53 Hatz K, Schneider U, Henrich PB, et al. Ranibizumab plus verteporfin photodynamic therapy in neovascular age-related macular degeneration: 12 months of retreatment and vision outcomes from a randomized study. Ophthalmologica 2015;233:66-73.

54 Csaky KG, Dugel PU, Pierce AJ, et al. Clinical evaluation of pazopanib eye drops versus ranibizumab intravitreal injections in subjects with neovascular age-related macular degeneration. Ophthalmology 2015;122:579-88.

55 Constable IJ, Pierce CM, Lai C-M, et al. Phase 2A randomized clinical trial: safety and post hoc analysis of subretinal rAAV. sFLT-1 for wet age-related macular degeneration. EBioMedicine 2016;14:168-75

56 Russo A, Scaroni N, Gambicorti E, et al. Combination of ranibizumab and indomethacin for neovascular age-related macular degeneration: randomized controlled trial. Clin Ophthalmol 2018;12:587-91.

57 Campochiaro PA, Marcus DM, Awh CC, et al. The Port delivery system with ranibizumab for neovascular age-related macular degeneration: results from the randomized phase 2 ladder clinical trial. Ophthalmology 2019;126:1141-54.

58 Comparison of Age-related Macular Degeneration Treatments Trials (CATT) Research Group, Martin DF, Maguire MG, et al. Ranibizumab and bevacizumab for treatment of neovascular age-related macular degeneration: two-year results. Ophthalmology 2012;119:1388-98.

59 Lim TH, Lai TYY, Takahashi K, et al. Comparison of ranibizumab with or without verteporfin photodynamic therapy for polypoida choroidal vasculopathy: the Everest II randomized clinical trial. JAMA Ophthalmol 2020;138:935-42. 